\title{
BRIGHTNESS DISTRIBUTION OF THE SUN AT 1.45 METRES
}

\author{
J. FIROR \\ Department of Terrestrial Magnetism, Carnegie Institution of Washington, \\ Washington, D.C., U.S.A.
}

The brightness distribution of the quiet sun at radio frequencies is of importance in determining the electron density and temperature in the chromosphere and the corona. Calculations made by Smerd, based on parameters derived from optical observations of the sun, indicate that the brightness distributions for wave-lengths near $\mathrm{I} \cdot 5$ metres are sensitive to the assumed coronal conditions and hence are well suited to checking these parameters.

The brightness distribution of the quiet sun at $\mathrm{I} \cdot 45$ metres has been derived from measurements made between December 1953 and July I 955 . The measurements were made with a variable-spacing interferometer and several fixed-spacing interferometers. The base-line of the interferometers was either east-west or north-south. With the fixed interferometers the method of measurement consisted of comparing the amplitude and phase of the output record produced by the sun with that produced by a known radio source-either the strong source in Cygnus or the Crab nebula. The measurements with the variable-spacing interferometer gave only the shape of the amplitude-spacing curve.

The measurements were made at times when the sun was quiet, as indicated by lack of burst activity, no large sunspots or new spot groups, and so on. The period during which measurements were made includes the epoch of sunspot minimum. The measurements vary, however, from day to day. In Fig. I the solid line gives the average amplitude of the interferometer trace as a function of spacing for the east-west base-line. The plotted points are the individual measurements and are seen to vary from the average curve. On any one day the measured points generally formed a smooth curve. The phase of the solar trace was always near zero for spacings up to about 100 wave-lengths and near $180^{\circ}$ for the region 100 to 15 wave-lengths. Only the amplitude was measured in the range 200 
to 340 wave-lengths, and in Fig. I these points are shown with assumed positive phase.

The results of the measurements made with the north-south base-line are shown in Fig. 2. In this case the maximum antenna spacing was smaller and no phase measurements were made. The overall similarity of the curve to that from the east-west measurements and the manner in which the measurements vary with spacing near 120 wave-lengths both indicate that the proper phase for the second maximum is negative, as plotted in Fig. 2.

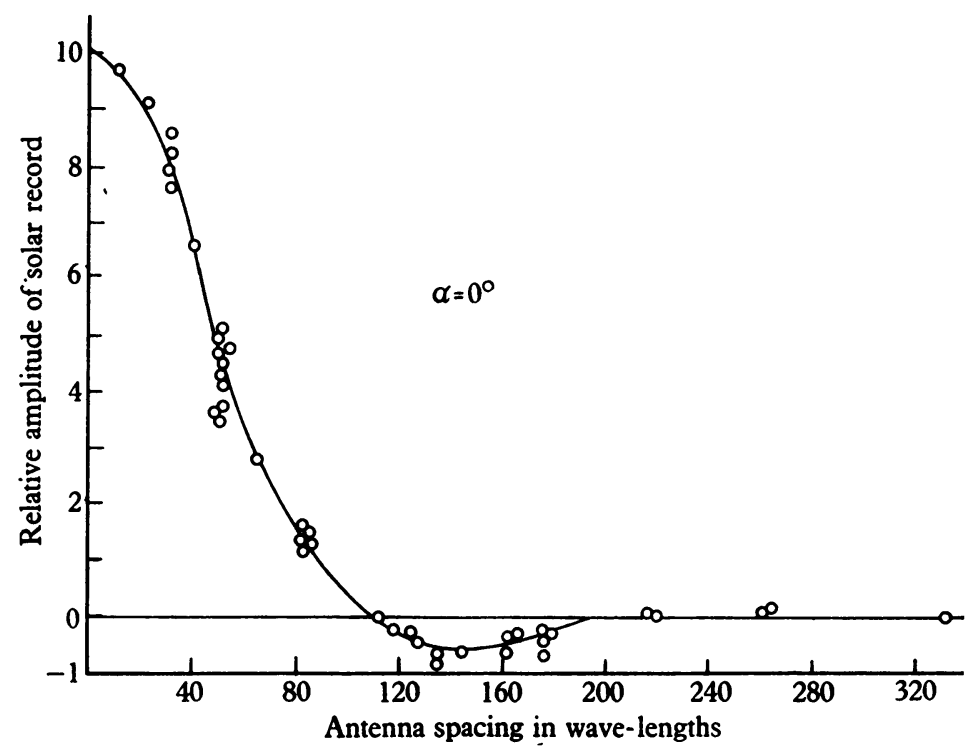

Fig. I. Amplitude of interferometer output as a function of antenna spacing for the case of the interferometer base-line parallel to the solar equator. The open circles are the measured values and the solid line is the average curve used for computing a brightness distribution. The negative values in the region of 100 to 200 wave-lengths indicate that, for these spacings, the interferometer output was a minimum at the time the centre of the solar disk was on the instrumental meridian.

A single curve, such as the one in Fig. I, can be used to derive a twodimensional brightness distribution if brightness contours of some particular form are assumed. With a second curve available, such as Fig. 2, the validity of the assumed contour shape can be partly checked and one parameter adjusted. If, for example, elliptical contours are assumed, the second experimental curve must be the same as the first with the abscissa expanded or compressed, and the parameter, which may be adjusted to fit the measured values, is the eccentricity of the elliptical contours.

For the present measurements it is seen that the north-south curve is not the same as the east-west curve, but has differences in shape rather than 
in scale. The brightness distribution must therefore have contours more complicated than central ellipses. The measurements are insufficient to determine these contours completely, but they may be reduced to give a distribution consistent with the measurements. This reduction was made by interpolation between the two measured curves to obtain curves for all inclinations of the interferometer fringes to the solar axis. The brightness distribution of the solar disk is then the two-dimensional Fourier transform of the set of amplitudes so obtained.

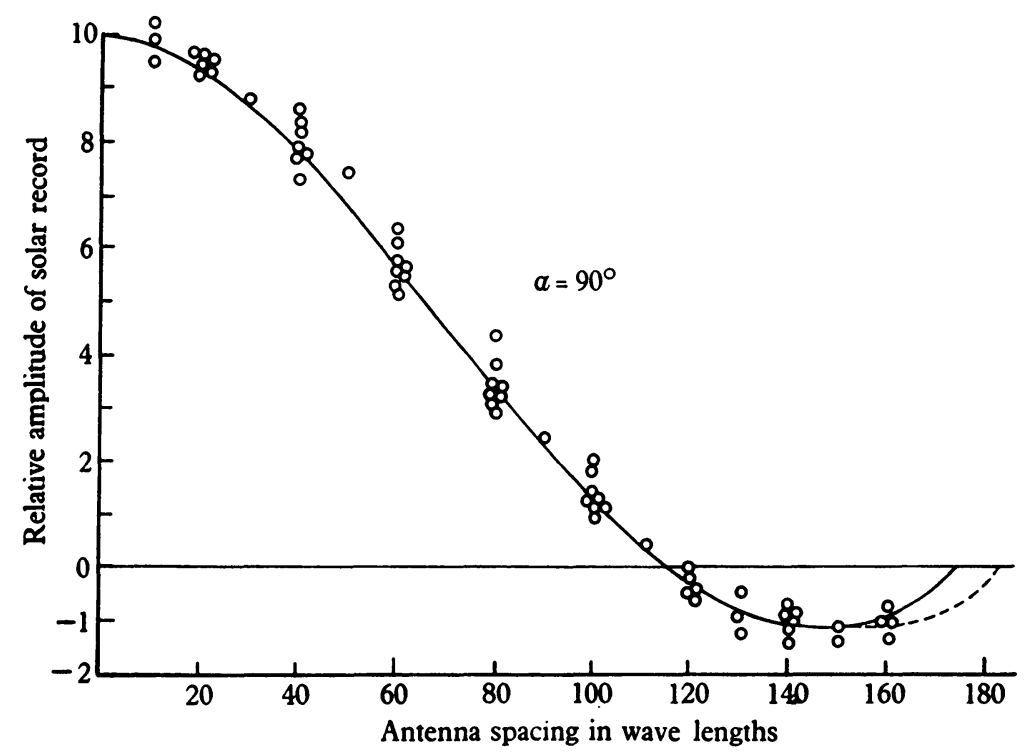

Fig. 2. Amplitude of interferometer output as a function of antenna spacing for the case of the interferometer base-line perpendicular to the solar equator.

The results of this procedure are given in the solid lines in Fig. $3 a$. The two curves are the brightness distributions along the solar equator and axis. It is seen that both curves exhibit the bright limb and that the equatorial curve is broader than the polar curve. Although both curves are quite similar to the $1 \cdot 5$-metre curve calculated by Smerd and shown in Fig. $3^{b}$, it must be remembered that we may add a great variety of distributions to the one obtained and still be entirely consistent with the experimental data. It is only necessary that the distributions to be added contain only higher frequencies, that is, distributions varying more rapidly with angle than are detected by the interferometer at its maximum spacing.

It is also to be noted that the derived brightness along the solar axis becomes negative around $\mathrm{I} \cdot 6$ solar radii. This is a result of the uncertainties 296 
in the amplitude of the interferometer trace at large spacings. For example, if the experimental curve in Fig. 2 is extended to zero in a different manner (dotted line) then the dotted curve in Fig. $3^{a}$ results, which does not become appreciably negative, but also has a more prominent bright limb. As we require a brightness distribution that does not become negative, the dotted line in Fig. 4 is perhaps the more reasonable one to associate with the present measurements.

Because the validity of the derived distribution was seen to depend so strongly on measurements at greater spacings, some points were taken at 216,260 and 340 wave-lengths with the east-west interferometer. The
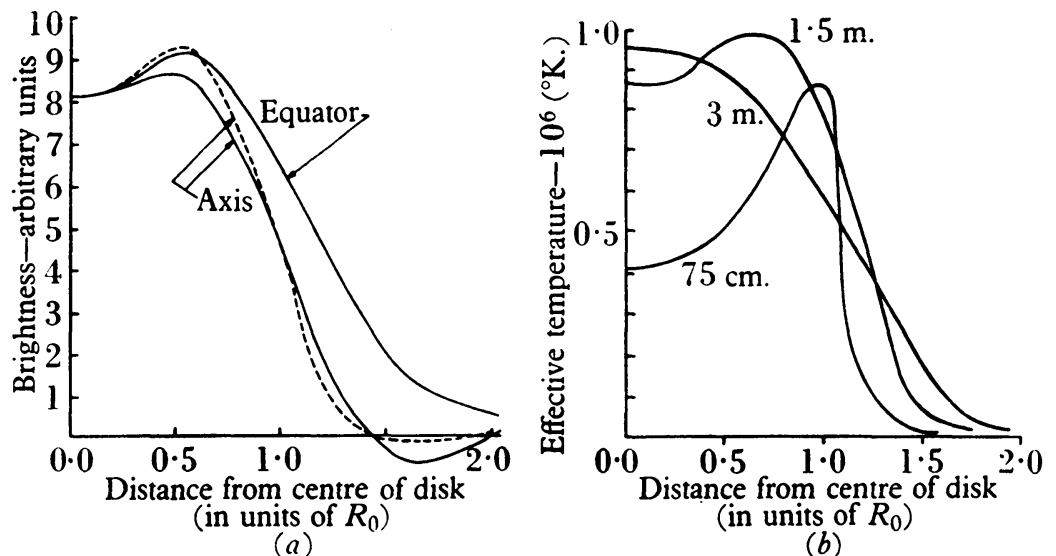

Fig. 3. (a) Brightness distribution of the sun at $1 \cdot 45$ metres wave-length. The solid curves are derived from the solid curves in Figs. I and 2. If the dashed curve in Fig. 2 is used, the equatorial curve is not changed appreciably while the brightness along the axis is changed to the dashed curve here. (b) Theoretical brightness distributions calculated by Smerd (1950).

amplitudes only were measured and were sufficiently small so that they do not change any major feature of the distribution derived without them, regardless of which phase is assumed. These points could, if taken into account, vary the details of the distribution, such as shifting the bright limb in or out a small amount, brightening or darkening the centre and so on. But the lack of any major change produced by these points lends confidence to the two main features of the derived distribution: the polar flattening and the bright limb.

\section{Discussion}

Unsöld: Why does the maximum brightness occur inside the limb?

Burke: This occurs also in some theoretical computations, e.g. Smerd's.

Pawsey: Moreover, aerial smoothing tends to move it inside. 